\title{
CONHECIMENTO DA POPULAÇÃO RELACIONADO À ASSISTÊNCIA DAS UNIDADES DE PRONTO ATENDIMENTO DE CURITIBA-PR
}

\author{
Amanda de Cassia Azevedo ${ }^{1}$ \\ André Luis Cândido da Silva ${ }^{1}$ \\ Suéllyn Mattos de Aragão ${ }^{1}$ \\ Helena Reback Graichen ${ }^{2}$ \\ Paulo de Carvalho Costa ${ }^{2}$ \\ Rafael Wind dos Santos ${ }^{2}$ \\ Solena Ziemer Kusma Fidalski ${ }^{1}$
}

AZEVEDO, A. de C.; SILVA, A. L. C. da; ARAGÃO, S. M. de; GRAICHEN, H. R.; COSTA, P. de C.; SANTOS, R. W. dos; FIDALSKI, S. Z. K. Conhecimento da população relacionado à assistência das unidades de pronto atendimento de Curitiba-Pr. Arquivos de Ciências da Saúde da UNIPAR, Umuarama, v. 25, n. 1, p, 3-10, jan./abr. 2021.

RESUMO: Introdução: As Unidades de Pronto Atendimento (UPAs) funcionam como porta de entrada para os casos de urgência e emergência e, muitas vezes, para casos de caráter não emergencial. Objetivo: Identificar o conhecimento da população a respeito dos serviços prestados pelas UPAs em Curitiba-PR. Método: Estudo observacional transversal, avaliou 516 pessoas que estavam em espera para atendimento nas UPAs, com classificação não urgente ou pouco urgente, segundo Protocolo Internacional de Manchester. A coleta se deu por meio de questionário semiestruturado aplicado por entrevistador. Resultados: $82,8 \%$ dos entrevistados acreditavam estar no local correto para o seu atendimento, fator que foi independente estatisticamente da escolaridade ou do conhecimento sobre as Unidades Básicas de Saúde (UBS); 86,6\% dos usuários foram à UPA por busca direta, e uma parcela de 24,9\% cogitou ir a uma UBS antes. Dentre os motivos mais frequentes de procura pela UPA, em ordem decrescente, abrangiam questões de locomoção, desconhecimento do funcionamento do sistema de saúde, motivos pessoais, a não necessidade de marcar consulta e, por fim, considerar a própria doença como grave. Conclusão: Existe a necessidade de se fortalecer o vínculo do usuário com as UBS, assim como uma melhor estruturação da atenção primária.

PALAVRAS-CHAVE: Serviços de Saúde de Emergência; Uso de Serviços de Saúde; Participação da Comunidade.

\section{KNOWLEDGE OF THE POPULATION REGARDING THE EMERGENCY CARE UNITS IN CURITIBA-PR}

ABSTRACT: Introduction: Emergency Care Units (ECU) work as a gateway for urgent and emergency cases and, often, also for nonemergency ones. Objective: Identification of the knowledge of the population regarding the services provided by the ECU in Curitiba-PR. Method: A cross-sectional observational study evaluated 516 people who were waiting for care in the ECU, classified as non-urgent or of little urgency, according to the Manchester International Protocol. Data collection occurred through a semi-structured questionnaire applied by an interviewer. Results: $82.8 \%$ of the interviewees believed they were in the correct place for their care, a factor that was statistically independent of schooling or knowledge about Basic Health Units (BHU); $86.6 \%$ of users went to the ECU by direct search, and $24.9 \%$ of users considered going to a BHU before. Among the most frequent reasons for going to the ECU, in decreasing order, ranged from locomotion, lack of knowledge about the operation of the health system, personal reasons, the possibility of just walking in, without the need to make an appointment and, finally, the fact that they considered the illness severe. Conclusion: There is a need to strengthen the user's bond with the BHU and consequently better structuring of primary care.

KEYWORDS: Emergency Health Services; Health Services; Community Participation.

Introdução

Problemas como a superlotação das Unidades de Pronto Atendimento (UPAs) e o acesso precário aos serviços de saúde de baixa, média e alta complexidade vêm aumentando consideravelmente nos últimos anos em todo o território nacional. A elevada procura pelos serviços de pronto atendimento ocorre concomitantemente à ausência de diálogo com a sociedade civil e limitação de gastos e investimentos voltados para o Sistema Único de Saúde (SUS) (MARIANO, 2017).

As UPAs foram instituídas pelo governo federal em novembro de 2002 como parte da Política Nacional de Urgência e Emergência, por meio da Portaria GM/MS 2048, com o objetivo de funcionar como porta de entrada para os casos de urgência ou emergência, podendo solucionar os problemas no local, encaminhar para hospitais ou mesmo redirecionar os pacientes a uma Unidade Básica de Saúde (UBS).
Em janeiro de 2017, por intermédio da Portaria GM/ MS 10/2017, houve a redefinição das diretrizes de modelo assistencial e financiamento das UPAs, contemplando questões relacionadas com o repasse de recursos ininterrupto, equipe assistencial multiprofissional com quantitativo de profissionais compatível com a necessidade do atendimento com qualidade, acolhimento e classificação de risco (UCHIMURA, 2015; BRASIL, 2002; BRASIL, 2017).

Curitiba foi uma das cidades pioneiras na criação de unidades de emergência pré-hospitalares fixas, servindo de inspiração para a criação das UPAs pelo governo federal, com iniciativas semelhantes nas cidades de CampinasSP, Belo Horizonte-MG e Rio de Janeiro-RJ (OLIVEIRA et al., 2015). Na ocasião do estudo, Curitiba contava com 110 unidades de saúde, 15 centros de atenção psicossocial (CAPS), 48 clínicas especializadas (ou ambulatórios de especialidades), 8 hospitais especializados, 15 hospitais gerais e 9 UPAs (CURITIBA, 2013; CURITIBA, 2015).

As UPAs organizam seu atendimento mediante

DOI: 10.25110 /arqsaude.v25i1.2021.7307

'Programa de Pós-Graduação em Saúde Coletiva, Setor de Ciências da Saúde, Universidade Federal do Paraná (UFPR), Curitiba (PR), Brasil. E-mail: amandaazevedo@ufpr.br

${ }^{2}$ Acadêmicos do Curso de Medicina da Pontifícia Universidade Católica do Paraná, Curitiba (PR), Brasil. 
protocolos técnico-científicos (GOMIDE et al., 2012). Conforme recomendado na portaria que instituiu as UPAs no país, as unidades de Curitiba adotam um sistema de triagem de pacientes. Triagem pode ter o sentido de classificar ou priorizar elementos, sendo que a classificação de risco não implica exclusão, e sim, compartimentação a partir de protocolos preestabelecidos. A perspectiva de acesso rápido ao atendimento em saúde é paulatina, mesmo que as unidades de saúde muitas vezes não possuam estrutura física, recursos humanos e equipamentos adequados para acolher tal demanda. (GILBOY et al, 2005). Há de se enfatizar que, conforme a lei do exercício profissional, o enfermeiro é o profissional competente para a realização da triagem (BRASIL, 2010).

A avaliação estratifica o quadro dos usuários desde emergência até não urgente, passando por estágios muito urgente, urgente e pouco urgente (MATOZINHOS et al., 2019). O sistema se fundamenta na utilização de cores para cada categoria, a saber: vermelha quando emergência, situação na qual o usuário necessita de atendimento imediato; laranja para muito urgente, quando ele necessita de atendimento o mais rápido possível; amarela na situação urgente, quando o paciente precisa ser avaliado, porém, já possui condições de aguardar o atendimento; e verde e azul, onde a situação é pouco urgente e não urgente, sendo que o usuário pode ser atendido na atenção básica, de forma ambulatorial (PINTO et al., 2012).

Profissionais de saúde destacam que a UPA, devido ao seu caráter curativo de curto prazo e atendimento 24 horas, vem sendo a opção dos pacientes, independentemente dos sintomas ou gravidade da enfermidade (SANTOS et al., 2014). Neste estudo, os autores enfatizam que boa parte dos usuários que procuravam as unidades tinham problemas que poderiam ter sido solucionados em UBS, deturpando o objetivo inicial de atendimento das UPAs.

Destaca-se que o aumento na demanda das unidades de emergência acaba gerando sobrecarga de trabalho para toda a equipe de funcionários, prejudicando a qualidade do serviço prestado e contribuindo para o aumento dos custos de atendimento (OLIVEIRA et al., 2015; SANTOS et al., 2014).

A procura pelas UPAs em situações não emergenciais foi, do mesmo modo, constatada em um relatório de gestão com dados referentes ao $3^{\circ}$ quadrimestre de 2015, lançado pela Secretaria de Saúde de Curitiba. Neste documento há, ademais, a apresentação dos números de uma pesquisa realizada no mesmo ano, que mostra que o tempo médio para atendimento em uma UPA na cidade era de uma hora e quarenta e dois minutos e que aproximadamente $28 \%$ dos atendimentos, demoraram mais de três horas para ocorrer. O relatório afirma que deve existir uma maior articulação com o Departamento de Atenção Primária para que ocorra uma melhoria nos fluxos de atendimento, evitando o aumento do tempo de espera e melhorando a qualidade dos atendimentos prestados (CURITIBA, 2015).

A atenção primária, porta de entrada no sistema de saúde, entre outras ações, é responsável pela promoção e recuperação da saúde e pelo acompanhamento de doenças crônicas, campanhas de vacinação, execução de programas de prevenção da saúde. As UPAs, que em conceito destinamse a atuar como centro resolutivo de atenção pré-hospitalar fixa, tendo como retaguarda hospitais de referência e apoio dos serviços móveis, acabaram por concentrar a demanda de atendimento geral da população (GARLET et al., 2009; MARQUES, LIMA, 2007; DRULA et al., 2008). Eventual desconhecimento da população pode resultar em um aumento da demanda de atendimentos nas UPAs e, da mesma forma, colocar em segundo plano as UBS (CURITIBA, 2015; GARLET et al., 2009)

Muitas vezes, mesmo para o atendimento de transtornos menores, os usuários buscam as UPAs por procura direta em vez de procurar a atenção básica por primeiro. Isso contribui com a superlotação do sistema de atendimento pré-hospitalar fixo, que é agravada ainda por problemas na infraestrutura, falta de profissionais e pelo sucateamento/ subfinanciamento do SUS. Tais fatores corroboram para dificultar o atendimento multiprofissional de qualidade.

Considerando as barreiras existentes para o acesso ao SUS, busca-se entender, a partir da perspectiva dos usuários, os motivos da procura pelo atendimento pré-hospitalar fixo em detrimento da atenção básica, mesmo em situações que não são caracterizadas como de urgência e/ou emergência. Diante deste contexto, o presente artigo tem como objetivo verificar o conhecimento da população a respeito dos serviços prestados pelas UPAs na cidade de Curitiba-PR, no período compreendido entre outubro de 2014 e julho de 2015.

\section{Metodologia}

Trata-se de um estudo observacional transversal. Para o desenvolvimento deste trabalho, os pesquisadores inicialmente entraram em contato com o responsável por cada uma das UPAs de Curitiba que estavam em atividade na época do estudo: 1) UPA Cajuru, 2) UPA Boqueirão, 3) UPA Fazendinha, 4) UPA Sítio Cercado, 5) UPA Boa Vista, 6) UPA Pinheirinho, 7) UPA Cidade Industrial de Curitiba (CIC) e 8) UPA Campo Comprido. O contato foi realizado para explicar a proposta da pesquisa, bem como agendar os dias da coleta de dados, a qual foi realizada em todas as unidades existentes.

A partir dos dados de frequência de atendimento em cada uma das UPAs, foram entrevistados 516 participantes. Definiu-se a população a ser estudada por meio de amostragem não probabilística, por conveniência (HULLEY et al., 2008). O público-alvo foi composto por indivíduos com idade igual ou superior a 18 anos, de ambos os sexos, que tenham procurado as UPAs de Curitiba e com capacidades físicas e mentais para responder a um questionário.

A coleta de dados foi realizada no período compreendido entre outubro de 2014 e julho de 2015, e ocorreu de maneira aleatória, sem estabelecimento de ordem de chegada do usuário à UPA, em todos os dias da semana, nos períodos manhã, tarde e noite. Inicialmente os usuários foram triados pela equipe de enfermagem local. Os usuários classificados nas categorias "pouco urgente" e "não urgente" (verde e azul, respectivamente), do protocolo internacional de Manchester, foram convidados a responder a um questionário. A coleta de dados realizou-se de maneira individualizada, em local reservado, nas próprias dependências da UPA.

O questionário semiestruturado, composto por 18 perguntas, foi elaborado pelos pesquisadores e as perguntas eram relacionadas à identificação pessoal (sexo, idade, 
escolaridade, estado civil, profissão), local de residência, reconhecimento de utilização de diferentes serviços de saúde e queixa atual de procura da UPA. Essa última questão foi disposta de forma não estruturada.

Os resultados foram organizados em planilha do Programa Computacional Microsoft Excel e depois analisados com o Programa Statistical Package for the Social Sciences (SPSS) versão 20.0. As variáveis qualitativas foram analisadas por intermédio de frequências absolutas e percentuais, e as variáveis quantitativas por meio de média, mediana, desvio padrão, valores mínimos e máximos. Para análise inferencial foi utilizado o teste do Qui-quadrado. Valores com $\mathrm{p}<0,05$ foram considerados significativos.

Ressalta-se que este projeto está em conformidade com as normas para pesquisa envolvendo seres humanos e foi aprovada pelo Comitê de Ética em Pesquisa da Pontifícia Universidade Católica do Paraná (CONEP Protocolo CAAE 35288914.5.0000.0020, número do parecer 779.543, data 04/09/2014).

\section{Resultados}

A amostra, composta por 516 pessoas, possuía média de idade de 36,4 anos e desvio padrão de 15,7 . O mais jovem paciente entrevistado possuía 18 anos na ocasião da pesquisa e, o mais idoso, 88 anos. A tabela 1 apresenta as características da população envolvida com o estudo.

Tabela 1: Caracterização da amostra.

\begin{tabular}{|c|c|c|c|}
\hline Variável & Níveis & $\mathbf{F}$ & $\%$ \\
\hline Sexo & $\begin{array}{l}\text { Feminino } \\
\text { Masculino }\end{array}$ & $\begin{array}{l}323 \\
193\end{array}$ & $\begin{array}{l}62,6 \\
37,4\end{array}$ \\
\hline Estado civil & $\begin{array}{c}\text { Casado } \\
\text { Divorciado } \\
\text { Solteiro } \\
\text { Viúvo }\end{array}$ & $\begin{array}{c}180 \\
41 \\
272 \\
23\end{array}$ & $\begin{array}{c}34,9 \\
7,9 \\
52,7 \\
4,5\end{array}$ \\
\hline Município de residência & $\begin{array}{c}\text { Curitiba } \\
\text { Região metropolitana } \\
\text { Outras cidades }\end{array}$ & $\begin{array}{c}447 \\
59 \\
10\end{array}$ & $\begin{array}{r}86,6 \\
11,5 \\
1,9\end{array}$ \\
\hline Escolaridade & $\begin{array}{l}\text { Nunca estudaram } \\
\text { Ensino fundamental incompleto } \\
\text { Ensino fundamental completo } \\
\text { Ensino médio incompleto } \\
\text { Ensino médio completo } \\
\text { Ensino superior incompleto } \\
\text { Ensino superior completo }\end{array}$ & $\begin{array}{c}7 \\
118 \\
51 \\
76 \\
184 \\
48 \\
32\end{array}$ & $\begin{array}{c}1,4 \\
22,9 \\
9,9 \\
14,8 \\
35,7 \\
9,3 \\
6\end{array}$ \\
\hline Possui plano de saúde sistema privado? & $\begin{array}{l}\text { Não } \\
\text { Sim }\end{array}$ & $\begin{array}{c}457 \\
59\end{array}$ & $\begin{array}{l}88,6 \\
11,4\end{array}$ \\
\hline Principal serviço de saúde utilizado & $\begin{array}{c}\text { SUS - Sistema público } \\
\text { Plano de Saúde - Sistema privado }\end{array}$ & $\begin{array}{c}494 \\
22\end{array}$ & $\begin{array}{r}95,7 \\
4,3\end{array}$ \\
\hline $\begin{array}{l}\text { Encaminhado por UBS ou outro serviço de } \\
\text { saúde? }\end{array}$ & $\begin{array}{l}\text { Não (procura direta) } \\
\text { Sim }\end{array}$ & $\begin{array}{c}447 \\
69\end{array}$ & $\begin{array}{l}86,6 \\
13,4\end{array}$ \\
\hline Conhece a UBS próxima à sua residência? & $\begin{array}{l}\text { Não } \\
\text { Sim }\end{array}$ & $\begin{array}{c}38 \\
478\end{array}$ & $\begin{array}{r}7,4 \\
92,6\end{array}$ \\
\hline Utiliza a UBS? & $\begin{array}{l}\text { Não } \\
\text { Sim }\end{array}$ & $\begin{array}{l}342 \\
174\end{array}$ & $\begin{array}{l}66,3 \\
33,7\end{array}$ \\
\hline Quais outros serviços de saúde utiliza? & $\begin{array}{c}\text { Unidade Básica de Saúde } \\
\text { Outras UPAs } \\
\text { Hospitais } \\
\text { Clínicas particulares (médicos, psicólogos, } \\
\text { fisioterapeutas ou outros) } \\
\text { Ambulatórios de especialidades } \\
\text { Outros } \\
\text { Nenhum }\end{array}$ & $\begin{array}{c}324 \\
346 \\
54 \\
18 \\
\\
18 \\
4 \\
12\end{array}$ & $\begin{array}{l}62,8 \\
67,1 \\
10,5 \\
3,5 \\
3,5 \\
0,8 \\
2,3\end{array}$ \\
\hline Frequência de utilização da UPA & $\begin{array}{c}\text { Primeira vez } \\
<3 \text { visitas ao ano } \\
3 \text { a } 6 \text { visitas ao ano } \\
7 \text { a } 12 \text { visitas ao ano } \\
>12 \text { visitas ao ano }\end{array}$ & $\begin{array}{c}58 \\
231 \\
165 \\
39 \\
23\end{array}$ & $\begin{array}{c}11,2 \\
44,8 \\
32 \\
7,6 \\
4,4\end{array}$ \\
\hline
\end{tabular}

Legenda: $\mathrm{F}$ = frequência absoluta; \% = percentual em função das respostas válidas. 
Dos 447 usuários que buscaram a UPA por procura direta, 23,7\% (106/447) pensaram em se dirigir a uma UBS antes.

A tabela 2 evidencia que não houve significância estatística $(p<0,05)$ entre pacientes que conhecem a UBS próxima a sua casa e os que não conhecem, quando analisado se os mesmos pensaram ou não em ir a uma UBS antes de procurar a UPA.

Tabela 2: Distribuição dos indivíduos de acordo com o conhecimento da existência de UBS próxima ao domicílio e a possibilidade de se dirigirem a uma UBS

\begin{tabular}{cccccccc}
\hline & $\begin{array}{c}\text { Conhecem UBS } \\
\text { próxima a sua casa }\end{array}$ & \multicolumn{2}{c}{$\begin{array}{c}\text { Não conhecem UBS } \\
\text { próxima a sua casa }\end{array}$} & \multicolumn{3}{c}{ Total } \\
Variável & $\mathbf{n}$ & $\mathbf{\%}$ & $\mathbf{n}$ & $\mathbf{\%}$ & $\mathbf{n}$ & $\mathbf{0}$ & $\mathbf{p}$ \\
\hline Pensaram em ir a uma UBS & 99 & 93,4 & 7 & 6,6 & 106 & 100 & 0,59 \\
Não pensaram em ir a uma UBS & 313 & 91,8 & 28 & 8,2 & 341 & 100 \\
\hline
\end{tabular}

Legenda: $\mathrm{F}=$ frequência absoluta; $\%=$ percentual em função das respostas válidas.

Quando questionadas se consideravam estar no local correto para o atendimento de seu problema de saúde atual, $82,8 \%(427 / 516)$ das pessoas responderam afirmativamente.
Essa predominância em considerar estar no local correto não sofreu diferença estatística, quando comparada entre os grupos de diferentes níveis de escolaridade (Tabela 3).

Tabela 3: Distribuição dos indivíduos de acordo com a concepção de que a UPA é o local correto para o atendimento do problema de saúde, e os diferentes níveis de escolaridade

\begin{tabular}{|c|c|c|c|c|c|c|c|}
\hline & $\begin{array}{l}\text { UPA é o local } \\
\text { correto de } \\
\text { atendimento da } \\
\text { queixa atual }\end{array}$ & & $\begin{array}{l}\text { UPA não é o } \\
\text { local correto de } \\
\text { atendimento da } \\
\text { queixa atual }\end{array}$ & & Total & & \\
\hline Escolaridade & $\mathbf{n}$ & $\%$ & $\mathbf{n}$ & $\%$ & $\mathbf{n}$ & $\%$ & $\mathbf{p}$ \\
\hline Nunca estudou & 7 & 1,4 & 0 & 0 & 7 & 1,4 & 0,18 \\
\hline Fundamental incompleto & 105 & 20,4 & 13 & 2,5 & 118 & 22,9 & \\
\hline Fundamental completo & 43 & 8,3 & 8 & 1,6 & 51 & 9,9 & \\
\hline Ensino médio incompleto & 65 & 12,6 & 11 & 2,1 & 76 & 14,7 & \\
\hline Ensino médio completo & 147 & 28,4 & 38 & 7,3 & 185 & 35,7 & \\
\hline Superior incompleto & 36 & 7 & 12 & 2,3 & 48 & 9,3 & \\
\hline Superior completo & 24 & 4,7 & 7 & 1,4 & 31 & 6,1 & \\
\hline Total $(n=516)$ & 427 & 82,8 & 89 & 17,2 & 516 & 100 & \\
\hline
\end{tabular}

Legenda: $\mathrm{F}=$ frequência absoluta; $\%=$ percentual em função das respostas válidas.

Analisando o grupo que considerou estar no local correto e que não pensou em ir a uma UBS, encontrou-se o resultado que corresponde a 52,1\% (269/516) do total da amostra. Enquanto que 4,1\% (21/516) pensou em ir na UBS e não considerou estar no local correto.

Dos 478 entrevistados que conheciam a UBS próxima a sua casa, $36,4 \%(174 / 478)$ realizavam programas rotineiros na unidade de saúde. Dessa mesma parcela da população, 82,8\% (396/478) considerava estar no local correto para o atendimento do seu problema de saúde atual. No grupo dos que não conheciam a UBS próxima de sua residência, 7,4\% (38/516), a porcentagem que julgava estar no local correto para o atendimento correspondeu a 76,3\% (29/38), proporcionando uma distribuição de frequências não estatisticamente significativa (Tabela 4).

Tabela 4: Comparação dos indivíduos de acordo com a concepção de que a UPA é o local correto para o atendimento do problema de saúde, com o conhecimento da UBS próxima aos seus lares.

\begin{tabular}{|c|c|c|c|c|c|c|c|}
\hline & $\begin{array}{l}\text { UPA é o local } \\
\text { correto de } \\
\text { atendimento da } \\
\text { queixa atual }\end{array}$ & & $\begin{array}{l}\text { UPA não é o } \\
\text { local correto de } \\
\text { atendimento da } \\
\text { queixa atual }\end{array}$ & & Total & & \\
\hline Variável & n & $\%$ & $\mathbf{n}$ & $\%$ & n & $\%$ & p \\
\hline $\begin{array}{c}\text { Conhecem UBS próxima a sua } \\
\text { casa }\end{array}$ & 396 & 93,4 & 82 & 6,6 & 478 & 100 & 0,31 \\
\hline $\begin{array}{c}\text { Não conhecem UBS próxima a } \\
\text { sua casa }\end{array}$ & 29 & 76,3 & 9 & 23,7 & 38 & 100 & \\
\hline
\end{tabular}

Legenda: $\mathrm{F}=$ frequência absoluta; \% = percentual em função das respostas válidas. 
Dentre as 174 pessoas que participavam de algum programa de acompanhamento ou faziam consultas rotineiras na UBS, $83,3 \%(145 / 174)$ avaliaram que estavam no local correto, de maneira similar aos 342 entrevistados que não faziam acompanhamento, dos quais $81,9 \% \quad(280 / 342)$ acreditavam estar no local correto, sem que se apresentasse um nível de confiança significativo ao se comparar estas variáveis (Tabela 5).

Tabela 5: Comparação dos indivíduos de acordo com a concepção de que a UPA é o local correto para o atendimento do problema de saúde, com o conhecimento da UBS próxima aos seus lares.

\begin{tabular}{|c|c|c|c|c|c|c|c|}
\hline & $\begin{array}{l}\text { UPA é o local } \\
\text { correto de } \\
\text { atendimento da } \\
\text { queixa atual }\end{array}$ & & $\begin{array}{l}\text { UPA não é o } \\
\text { local correto de } \\
\text { atendimento da } \\
\text { queixa atual }\end{array}$ & & Total & & \\
\hline Variável & $\mathbf{n}$ & $\%$ & $\mathbf{n}$ & $\%$ & $\mathbf{n}$ & $\%$ & $\mathbf{p}$ \\
\hline Frequenta uma UBS & 145 & 83,3 & 29 & 16,7 & 174 & 100 & 0,68 \\
\hline Não frequenta UBS & 280 & 81,9 & 62 & 18,1 & 342 & 100 & \\
\hline
\end{tabular}

Legenda: $\mathrm{F}=$ frequência absoluta; \% = percentual em função das respostas válidas.

Na figura 1, é possível verificar qual o principal motivo assinalado para a procura da UPA:

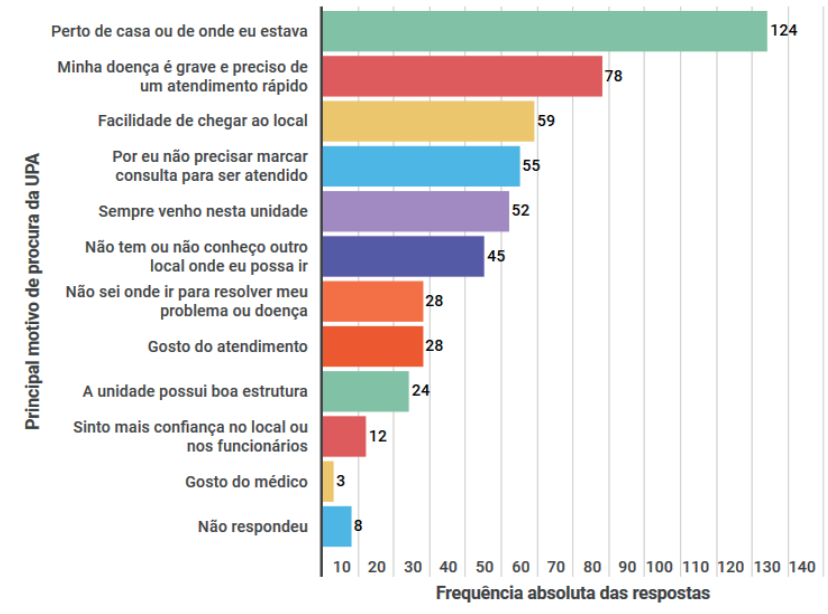

Figura 1: Principal motivo de procura da UPA.

De todos os entrevistados, 7,4\% (38/516) referiram não conhecer a UBS próxima de sua casa. Em comparação, aqueles que responderam ter como principal motivo de busca da UPA "não ter outro lugar para ir ou não conhecer outro lugar que possa ir", 17,8\% (8/45) relataram não conhecer a UBS. De maneira semelhante, do grupo que respondeu como principal motivo não saber onde ir para resolver seu problema ou doença, 14,3\% (4/28) não conhecem a UBS.

Os principais motivos que levaram os 23 pacientes que frequentam mais de 12 vezes por ano a UPA foram: achar que a doença era grave e necessitar de um atendimento rápido, com 21,7\% (5/23); e por não precisar marcar consulta para ser atendido também com 21,7\% (5/23). Em contrapartida, quanto aos pacientes que frequentavam a UPA até 6 vezes por ano, para $24,5 \%(111 / 454)$, o principal motivo de busca de atendimento era por ser perto do local onde estava.

Analisando os sintomas mais comuns relatados pelos participantes da pesquisa observou-se que a "dor de cabeça" foi o mais prevalente, com 88 citações. Esta análise também considerou aqueles pacientes que citaram mais de uma queixa $(26,6 \% ; 137 / 516)$ como motivo da busca pela unidade. As outras queixas mais citadas, em ordem de frequência, foram: "febre" (n=49), "gripe" $(n=47)$, "dor de barriga" (n=38), “dor nas costas" $(n=32)$, “dor de garganta" $(n=32)$, "diarreia" e "vômitos" $(n=26)$, sintomas respiratórios $(n=25)$ e sintomas oculares $(n=18)$.

\section{Discussão}

Frequentemente são encontrados nas UPAs usuários cujas condições clínicas não correspondem a real vocação do serviço. Este fato acaba por gerar um fluxo que excede as capacidades de atendimento do local (GARLET et al., 2009). Muito embora as queixas mais encontradas também possam estar presentes em situações emergenciais, nestes casos foram classificadas como não urgentes pelo protocolo de Manchester.

Apesar dos sintomas não serem urgentes, 82,8\% dos entrevistados acreditavam estar no local correto, quando efetivamente, deveriam ser atendidos em uma UBS. Nesse sentido, não se pode deixar de mencionar os fatores de mudança de sintoma do usuário até o momento da avaliação, ou até mesmo um equívoco no momento da avaliação e classificação de risco. Deve-se notar que os diferentes níveis de escolaridade não foram determinantes nessa questão. Tal resultado também não se referiu ao desconhecimento, pelo público, das UBS, visto que 92,6\% (478/516) relataram conhecê-las. Uma porcentagem igualmente alta de conhecimento foi encontrada tanto entre aqueles que pensaram quanto entre os que não pensaram em ir antes à UBS. Uma provável razão para isso foi a falta de vínculo entre a UBS e a população de seu distrito sanitário, observado pela grande parcela $(66,3 \% ; 342 / 516)$ que não realizava acompanhamento ou fazia consultas rotineiras no serviço primário. Conclui-se que grande parte da população que acessou a UPA, apesar de conhecer a unidade de atenção primária, não a frequentava. Uma das limitações do estudo diz respeito à idade dos entrevistados, pois mais de 50\% dos usuários têm menos de 40 anos e, portanto, não apresentam comorbidades, fazendo com que esse segmento de usuários não possua o hábito de buscar os serviços da atenção básica.

Dentro o grupo $(33,7 \% ; 174 / 516)$ que frequentava a UBS e buscou assistência na UPA, 13,4\% (69/516) das pessoas foram encaminhadas, mesmo posteriormente tendo sido triadas como não urgentes pelo protocolo de Manchester. É possível que as UBS não tenham conseguido assistir esses usuários devido à equipe multiprofissional de saúde subdimensionada, falta de medicamentos, falta de 
equipamentos para realização de exames complementares, superlotação da agenda de atendimentos das UBS, ou mesmo um erro de triagem ou diagnóstico, tanto por parte do atendimento primário quanto da UPA que recebeu o paciente (MOURA et al., 2010).

De acordo com Cremonesi et al. (2015), a superlotação é um dos problemas mais prejudiciais à gestão de serviços de emergência. Pesquisa realizada em um grande hospital italiano constatou que o uso de um sistema de triagem classificatória de risco robusto implica em correlação positiva entre a superlotação e o tempo de espera de pacientes não urgentes, sem influência no tempo de atendimento de casos urgentes.

A presente pesquisa mostrou que, dos $86,6 \%$ dos entrevistados que não foram encaminhados, apenas $24,9 \%$ cogitou buscar antes uma UBS. Isso indica que, para a população, a UPA era a opção de resolutividade de doenças independentemente do seu grau de gravidade, afastando as UBS a um papel secundário.

Segundo Guedes et al. (2013), esse tipo de informação nem sempre está presente na maioria das fichas de atendimento, mas consiste em um dado importante para a análise da integração entre os diferentes componentes da rede de atenção à urgência proposto no país, além de apontar o grau de orientação da população quanto à acessibilidade e objetivos de cada serviço inserido nesse tipo de configuração. Para tentar entender o porquê dessa troca de função por parte da população, realizou-se indagação do principal motivo de busca da unidade naquele momento. Foi constatado que os motivos que margeiam questões de comodidade ou locomoção são os mais significativos $(45,5 \%)$, tais como: "sempre venho nessa unidade", "facilidade de chegar ao local" e "perto de casa ou do lugar que estava". As UPAs apresentavam uma localização geográfica privilegiada, normalmente próximas de terminais de ônibus, sendo que esse fato se identificou como mais importante na escolha de qual serviço utilizar, em detrimento do estado de saúde.

Pode-se inferir que boa parte da população desconhecia o fluxo do sistema, conforme verificado pela porcentagem de $14,1 \%$ de pessoas que referiram não saber onde ir ou não ter outro local para resolver seu problema. Observou-se que, dentro desse grupo, o desconhecimento da UBS próxima de sua casa foi mais do que 2 vezes maior quando comparado a amostra total. Para essa população, medidas de conscientização fazem-se necessárias e podem ser efetivas para desafogar o sistema.

Um outro grupo de motivos (13\%) denota, inclusive, falta de vínculo com a UBS, ou uma falta de estrutura da atenção básica como um todo. O Relatório de Gestão que contempla informações referentes ao $3^{\circ}$ Quadrimestre de 2015, mostra que, em outubro do referido ano, a cobertura da atenção básica estava em $58,9 \%$. Significa que existiam vazios assistenciais no município ou unidades de saúde trabalhando acima da capacidade instalada. Respostas como "gosto do médico", "gosto do atendimento", "a unidade possui boa estrutura" e "sinto mais confiança no local ou nos funcionários", mostram que essas pessoas têm o hábito de frequentar UPA por motivos pessoais, e não necessariamente por sua função real. Dentro deste grupo, a proporção de pessoas que consideraram estar no local correto foi maior do que da amostra total.
A falta de necessidade de marcar consulta para ser atendido, de maneira isolada, correspondeu a 10,7\% das respostas dos entrevistados, elencando a quarta posição de maior frequência. Esse fato pode decorrer de uma carência na capacidade do sistema primário de suportar a demanda da população, do tempo prolongado para conseguir uma consulta médica, ou ainda um número insuficiente de profissionais da saúde. Deve-se considerar ainda, que os horários de atendimento das UBS são menos abrangentes em comparação com as UPAs, uma vez que a maioria delas não abre aos sábados, domingos, feriados e períodos noturnos.

Segundo os dados coletados, a maior parte da população usuária das UPAs a utilizava menos de 3 vezes ao ano. Dentre os que frequentavam mais de 12 vezes por ano, o motivo encontrado para a busca difere do principal motivo da amostra geral, sendo no primeiro grupo a não necessidade de agendamento prévio o fator decisivo. Isso comprova que uma correção no sistema de funcionamento das UBS deslocaria essa parcela que muito utiliza os prontos atendimentos para o serviço de atenção básica.

Desde 2011 o Ministério da Saúde preconiza uma atuação em "Rede" dos serviços de saúde. As redes correspondem à articulação entre os diferentes serviços que o SUS oferece (unidades de saúde, UPA, CAPS) e sistemas de saúde e às relações entre os profissionais atuantes, mediante relações de interdependência entre os pontos da Rede. A articulação desses serviços é primordial para que o usuário tenha suas necessidades atendidas, garantindo assim, a melhoria do acesso, da qualidade, da resolubilidade e da integralidade do cuidado. A efetividade da rede de atenção depende da compreensão e apoio dos usuários do sistema para com os fluxos e articulação da rede (BRASIL, 2014).

Mas ainda não é tudo. São tempos de cuidado para toda rede. A Emenda Constitucional 95/16 congela os gastos públicos por vinte anos, sem considerar as mudanças demográficas, epidemiológicas e a necessidade de incorporação tecnológica do SUS. Fere de morte o SUS e desconsidera as necessidades de saúde da população, de expansão da rede para cobrir vazios assistenciais e a própria inflação setorial. Pior, desvincula os gastos sociais de qualquer crescimento de receitas nos próximos 20 anos (REIS et al., 2016). Tornam inviáveis programas como o Mais Médicos, as Unidades de Pronto Atendimento (UPAs), o Serviço de Atendimento Móvel de Urgência (Samu), a Farmácia Popular, a Saúde da Família, o Programa Nacional de Imunizações, entre outros. As reformas impostas destroem políticas universalizantes e inclusivas que foram construídas nos últimos anos.

De acordo com os princípios do SUS, bem como com as políticas públicas, que orientam a organização dos serviços, a porta de entrada da atenção básica pode ser representada pelas UBS. Deve ser observado o fato de que a população necessita ser orientada para bem utilizar os serviços do SUS, de forma que a população aprenda sobre o uso dos serviços da rede, o que inclui atenção básica e a UPA, conforme suas necessidades, e não de acordo com suas preferências.

Embora este estudo tenha buscado identificar o conhecimento da população em relação a utilização das UPAs, o modelo de estudo transversal aplicado nesta pesquisa apresentou algumas fragilidades, como o fato de a 
coleta não ocorrer de maneira equitativa entre os períodos do dia e da semana e a possível falta de entendimento do questionário por parte dos participantes do estudo. Também há que se destacar que a coleta de dados ocorreu em um único momento e que há dificuldades de se investigar as condições que apresentaram baixa prevalência, considerando que seria necessária uma amostra relativamente maior (BASTOS; DUQUIA, 2007). Também não é possível prever se os fatores de confusão se distribuem de forma igual entre os diferentes grupos (características socioeconômicas, por exemplo) envolvidos com a pesquisa.

\section{Considerações Finais}

As UBS, como porta de entrada dos serviços de saúde do SUS, por inúmeros motivos, entre eles, por possuírem profissionais com maior tempo de trabalho naquela área, menor rotatividade de recursos humanos, serem referência territorial de saúde, incumbir-se como unidade de saúde mais próxima da residência do usuário, deveriam estabelecer um vínculo maior com a clientela e ser parâmetro de primeira escolha para ações de promoção, prevenção e proteção de saúde em caso de necessidade, excetuando os casos de urgência e emergência. Entretanto, esses argumentos não são suficientes para evitar que muitos usuários utilizem as Unidades de Pronto Atendimento como entrada preferencial na iminência de sintomas que poderiam ser avaliados e tratados na atenção básica.

Cabe salientar que, para a atenção básica se tornar a porta de entrada preferencial do usuário e a responsável de fato pelo cuidado, alguns aspectos relacionados à gestão de recursos humanos e também à melhoria do acesso devem ser considerados. Importante destacar a necessidade de educação permanente e continuada da equipe de saúde a respeito do acolhimento, acesso, triagem, educação em saúde com informação constante ao usuário nas consultas de enfermagem, médica, nas atividades de odontologia e desempenhadas pelo auxiliar de enfermagem sobre o horário de funcionamento das unidades básicas de saúde e UPA, a diferença entre elas, e o porquê dos encaminhamentos para as Unidades de Pronto Atendimento.

Há que se ressaltar que as UBS sofrem, muitas vezes, com a falta de infraestrutura, falta de insumos, de medicamentos e de profissionais da saúde, o que leva a população a procurar as UPAs de imediato. Além disso, existe a restrição de horário das unidades básicas de saúde, em contrapartida das 24 horas de funcionamento das UPAs, favorecendo grande parte da população que trabalha e precisa de atendimento. De qualquer forma, deve-se compreender a finalidade e a diferença dos dois serviços, que pertencem a níveis distintos de atenção à saúde. $\mathrm{O}$ respeito a esses parâmetros, atrelado à melhoria de condições de atendimento ao usuário nas unidades básica de saúde formam, juntos, o ponto de partida para o restabelecimento da utilização adequada dos usuários das UPAs.

\section{Referências}

BASTOS, J. L. D.; DUQUIA, R. P. Um dos delineamentos mais empregados em epidemiologia: estudo transversal. Sci. Med. v. 17, n. 4, p. 229-232, 2007.
BRASIL. Conselho Regional de Enfermagem do Distrito Federal. Parecer Técnico n⿳0 005/2010. Brasília (DF) 2010. Disponível em: https://www.coren-df.gov.br/site/ no-0052010-atribuicao-do-profissional-de-enfermagem-natriagem-com-classificacao-de-risco-nos/. Acesso em: 20 fev. 2020 .

BRASIL. Ministério da Saúde. Portaria GM/MS n. 2048, de 5 de novembro de 2002. Aprova o Regulamento Técnico dos Sistemas Estaduais de Urgência e Emergência. Diário Oficial da União, 12 nov. 2002; Seção 1: 32-54. Disponível em: https://www.jusbrasil.com.br/diarios/739297/pg-32secao-1-diario-oficial-da-uniao-dou-de-12-11-2002. Acesso em: 20 fev. 2020.

BRASIL. Ministério da Saúde. Portaria GM/MS n.10, de 3 de janeiro de 2017. Redefine as diretrizes de modelo assistencial e financiamento de UPA 24h de Pronto Atendimento como Componente da Rede de Atenção às Urgências, no âmbito do Sistema Único de Saúde. Diário Oficial da União, 4 jan. 2017; Seção 1:34-37. Disponível em: https://www.jusbrasil.com.br/diarios/134056179/dousecao-1-04-01-2017-pg-34. Acesso em: Acesso em: 20 fev. 2020 .

BRASIL. Ministério da Saúde. Secretaria de Atenção à Saúde. Implantação das Redes de Atenção à Saúde e outras Estratégias da SAS. Brasília. 2014. 160 p.

CREMONESI, P. et al. The robustness and effectiveness of the triage system at times of overcrowding and the extra costs due to inappropriate use of Emergency Departments. Appl. Health Econ. Health Policy, v. 13, n. 5, p. 507-514, 2015.

CURITIBA. Secretaria Municipal de Saúde. Gestão 2015 - Secretaria Municipal de Saúde. Relatório de gestão: monitoramento quadrimestral do SUS - Terceiro quadrimestre. Curitiba: Prefeitura Municipal de Curitiba, 2015.

CURITIBA. Secretaria Municipal de Saúde. Plano Municipal 2013 - Secretaria Municipal de Saúde. Plano municipal de saúde - 2010/2013. Curitiba: Prefeitura Municipal de Curitiba, 2013.

DRULA, K. D.; OLIVEIRA, R. A. de J.; MAGALHÃES, L. B. Diagnósticos de enfermagem no Centro Municipal de Urgências Médicas Boqueirão: utilização da Classificação Internacional para a Prática de Enfermagem. Boletim de Enfermagem, v. 1, n. 2, 2008.

GARLET, E. R. et al. Finalidade do trabalho em urgências e emergências: concepções de profissionais. Rev. Latino-Am. Enfermagem, v. 17, n. 4, p. 535-540, 2009.

GILBOY, N. et al. Emergency Severity Index, Version 4: implementation handbook. agency for healthcare research and quality. May 2005.

GOMIDE, M. F. S.; PINTO, I. C.; FIGUEIREDO, L. A. 
Acessibilidade e demanda em uma Unidade de Pronto Atendimento: perspectiva do usuário. Acta Paul. Enferm. v. 25, p. 19-25, 2012.

GUEDES, M. V. C.; HENRIQUES, A. C. P. T.; LIMA, M. M. N. Acolhimento em um serviço de emergência: percepção dos usuários. Rev. Bras. Enferm. v. 66, n. 1, p. 31-37, 2013.

HULLEY, S. B.; CUMMINGS, S. R.; BROWNER, W. S. et. al. Delineando a pesquisa clínica: uma abordagem epidemiológica. 3. ed. Porto Alegre : Artmed, 2008. 384 p.

MARIANO, C. M. Emenda constitucional 95/2016 e o teto dos gastos públicos: Brasil de volta ao estado de exceção econômico e ao capitalismo do desastre. Rev. Investig.

Constituc. v. 4, n. 1, p. 259-281, 2017.

MARQUES, G. Q.; LIMA, M. A. S. Demandas de usuários a um serviço de pronto atendimento e seu acolhimento ao sistema de saúde. Rev. Latino-Am. Enfermagem, v. 15, n. 1, p. 1-8, 2007.

MATOZINHOS, F. P. et al. Análise da triagem e dos atendimentos a mulheres vítimas de acidentes de trânsito. Rev. Bras. Enferm. v. 72, n. 4, p. 1070, 1076, 2019.

MOURA, B. L. A. et al. Atenção primária à saúde: estrutura das unidades como componente da atenção à saúde. Rev. Bras. Saúde Mater. Infant. v. 10, n. 1, p. 569-581, 2010.

OLIVEIRA, S. N. et al. Unidade de Pronto Atendimento - UPA 24h: percepção da enfermagem. Texto Contexto Enferm. v. 24, n. 1, p. 238-244, 2015.

PINTO, D. J.; SALGADO, P. O.; CHIANCA, T. C. M. Validade preditiva do Protocolo de Classificação de Risco de Manchester: avaliação da evolução dos pacientes admitidos em um pronto atendimento. Rev. Latino-Am. Enfermagem, v. 20, n. 6, p. 1041-1047, 2012.

REIS, A. A. C. et al. Tudo a temer: financiamento, relação público e privado e o futuro do SUS. Saúde em Debate, v. 40, n. especial, p. 122-135, 2016.

SANTOS, J. L. G. et al. Contexto organizacional e gerência do cuidado pelos enfermeiros em unidades de pronto atendimento. Rev. Gaúch. Enferm. v. 35, n. 4, p. 58-64, 2014.

UCHIMURA, L. Y. T. et al. Unidades de Pronto Atendimento (UPAs): características da gestão às Redes de Atenção no Paraná. Saúde em Debate, v. 39, n. 107, p. $972-$ 983, 2015. 